\title{
Bilateral Spontaneous Chronic Subdural Heamatoma Masquerading As Chronic Headache- A Case Report.
}

\author{
T.P. Mbaave ${ }^{1}$, G.I. Achinge ${ }^{1}$, H.Mohammad ${ }^{2}$, S.A.Obekpa ${ }^{1}$. \\ ${ }^{1}$ Department of Medicine, ${ }^{2}$ Department of Radiology, Benue State University \\ Teaching Hospital Makurdi, Nigeria.
}

\begin{abstract}
Background: A chronic subdural hematoma (CSDH) is defined as chronic ( $\geq 3$ weeks) intracranial bleeding between the dura mater (which adheres to the skull), and the arachnoid mater which envelops the brain. It is usually traumatic and unilateral but rarely, spontaneous hematoma occurs especially in the elderly. Both could be bilateral. The neurological symptoms are usually non specific and may not be readily recognized in the absence of a clear history of head trauma. This is more so in resource limited environments where there is paucity of diagnostic facilities like the computerized tomography (CT) scan. We present a case of spontaneous bilateral acute on chronic hematoma masquerading as chronic headache in a 56 year old man.
\end{abstract}

Method: The Case file of a 56 year old man admitted to the medical ward of the benue state university teaching hospital with chronic headache as well as literature search on the subject were used.

Result: A 56 year old man was admitted with chronic headache and neurological deficits without any history of head trauma. He was found to have bilateral chronic subdural hematoma on CT scanning of the brain with one side showing acute on chronic hemorrhage and was managed conservatively with good outcome.

Conclusion: Spontaneous chronic hematoma should be considered early in the diagnosis of chronic headache for improved patient outcomes.

Keywords: subdural, hematoma, acute, chronic, headache

\section{Introduction}

A chronic subdural hematoma (cSDH) is defined as chronic ( $\geq 3$ weeks) intracranial bleeding between the dura mater (which adheres to the skull), and the arachnoid mater (which envelops the brain). It may act as a space occupying lesion causing raised intracranial pressure and cerebral compression with attendant neurological sequel. The underlying cause of $\mathrm{cSDH}$ is usually traumatic tearing of the bridging veins which connect the brain surface with the dura mater. The incidence of cSDH is estimated at 1.7-18 per 100'000 people. The most common risk factor is head trauma ${ }^{1,2,3}$. Spontaneous hematoma also occurs especially with advanced age, and other risk factors such as alcohol abuse, seizures, cerebrospinal fluid (CSF) shunts, coagulopathies, anticoagulants, and patients at risk for falling (e.g. hemiplegia).Activities that increase intracranial pressure like straining, cough, blowing and weight lifting have also been found to be risks for spontaneous cSDH. In $20-25 \%$ of the cases, cSDHs are bilateral ${ }^{1,4}$. The incidence of cSDH is expected to rise worldwide with increasing population of elderly people. With the of availability of CT scan machines in some centers in Nigeria there has an observed increase in cases of cSDH. ${ }^{5,6}$. The neurological symptoms of chronic subdural hematoma are non specific such as headache which is the commonest symptom, altered mental status, disturbance of gait. Variable neurological deficits, hemiparesis and coma may be present in large hematomas ${ }^{3,4,6}$.It may not be readily recognized in the absence of a clear history of head trauma ${ }^{2,7,8}$. This is more so in our environment where there is paucity of diagnostic facilities like the CT scan as only some tertially health centers have these machines. Thus there is need for high index of suspicion in order to make the diagnosis.

\section{Case Report}

Mr. A.A, a 56year old health worker, was admitted to the medical ward of the Benue State University Teaching Hospital (BSUTH) with a two months history of headache. . The headache was mainly frontooccipital, throbbing and got progressively worse. He had mild neck pain but no dizziness, fainting spells, photophobia, vomiting, blurred vision, fever or other symptoms until the week before presentation when he felt increasingly weak Review of systems was not contributory. He had visited several hospitals and consumed several doses of antibiotics and analgesics since onset of the headache without any relief.

Few days prior to the onset of the headache he had upper respiratory tract infection (URTI) with profuse sneezing bouts and nasal blockade. He was hypertensive controlled on Moduretic and Amlodipine . He also had a history of Bell's palsy five years earlier and was HIV positive on highly active antiretroviral therapy 
(HAART). He did not consume alcohol, smoke cigarette or use recreational drugs and was married with children.

On examination he was a middle aged man who was conscious and oriented, afebrile, not pale, anicteric. The nasal turbinates were not engorged. Pulse was 86/minute regular and full volume, Blood pressure $140 / 90 \mathrm{mmHg}$ with normal cardiac apex, and heart sounds. Respiratory and abdominal examination findings were normal. He was fully conscious with Glasgow Coma Score (GCS) of 15/15. Neck rigidity and Kernig's sign were negative. He had normal gait. Pupil size and reaction, funduscopy and other cranial nerves were normal while he had normal tone and full power in all limbs.

A working diagnosis of chronic headache of uncertain cause was made with differentials of brain tumor, chronic asceptic meningitis and sinusitis. The patient was maintained on his HAART, antihypertensives, paracaetamol $1 \mathrm{~g}$, tds and intravenous ceftriazone $2 \mathrm{~g}, 12$ hourly pending definitive diagnosis.

On the 3rd admission day he as noted to be excessively sleepy with dizziness and unsteady gait when he attempted to walk. He also had a focal seizure on the 4th admission day. Neurological examination findings were otherwise same. Carbamazepine $200 \mathrm{mg}$ b.d was added to his medications. His Full Blood Count (FBC) revealed normal Hemoglobin of $12.5 \mathrm{~g} / \mathrm{dl}$; White Blood Count (WBC) of $4.6 \times 10^{3} / \mu \mathrm{L}$ with normal differentials (47\%lymphocytes, $40 \%$ neutrophils), and Thrombocytopenia with a Platelet count of $64 \times 10^{3} \mu \mathrm{L}$. Electrolytes, urea and creatinine levels and urinalysis were normal. Abdominal scan showed normal organs. A CT scan of the brain obtained on the 4th admission day showed a right chronic and a left acute on chronic subdural haematoma. (fig.1).

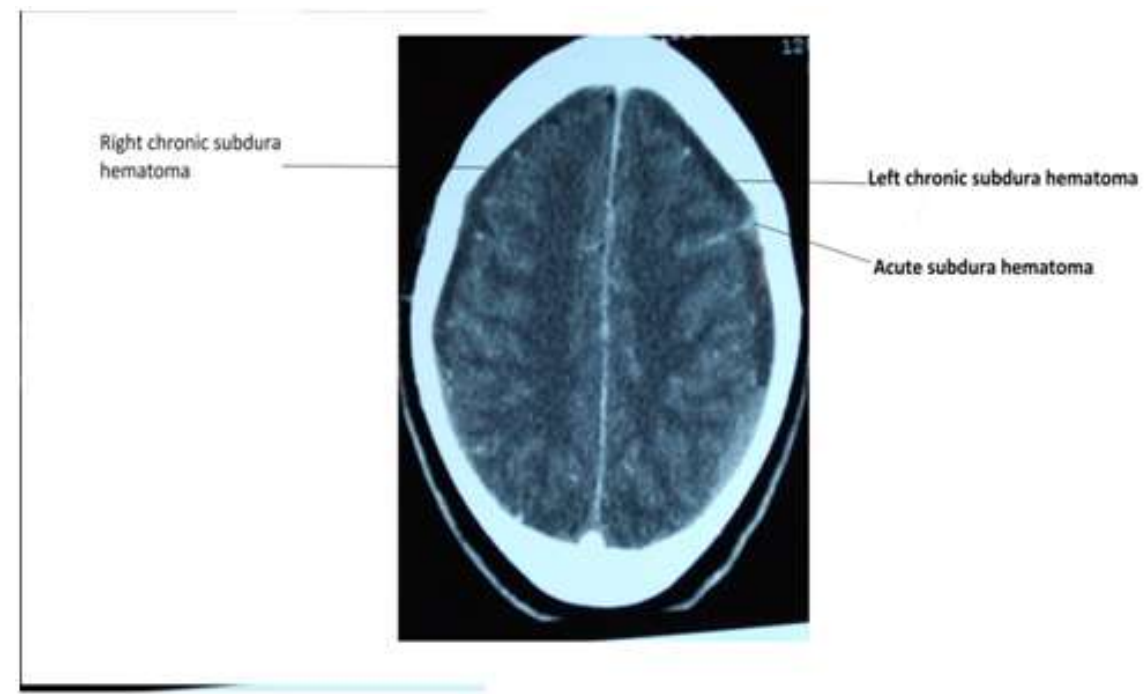

Fig.1. Axial CT scan of the patient at the level of the vertex showing bilateral hypo dense concavoconvex areas sub adjacent to the cranial vault. The left also shows a hyper dense area consistent with an acute bleed.

He was placed on Intravenous Dexamethasone 8mg, 8hourly and Intramuscular Tramadol 100mg 12hourly. A neurosurgical review advised conservative management on observation of a surgically insignificant hematoma and clinical improvement of the patient with commencement of steroids. Dexamethazone and Tramadol were converted to oral forms. His headache gradually subsided and he thereafter made an uneventful recovery and was discharged to the medical outpatient clinic on the $10^{\text {th }}$ day of admission. He was last seen in the clinic 2 weeks after discharge and was doing well.

\section{Discussion}

Spontaneous or non traumatic hematomas are rare. It is more in adults in the $5^{\text {th }}$ to $7^{\text {th }}$ decade of their lives ${ }^{7,8}$. Our patient was a 56 yr old man (in the $6^{\text {th }}$ decade of his life) whose complaint for most of the duration of his illness was headache. Before the result of the CT scan, the diagnosis of subdural hematoma was apparently not considered in all the health facilities he attended including our hospital. This is consistent with other reports where at presentation, the diagnosis was not considered because of the absence of clear history of trauma ${ }^{6,9,10}$.The patient had severe sneezing episodes prior to onset of symptoms, thrombocytopenia, hypertension and was HIV positive all of which are documented risk factors associated with spontaneous cSDH ${ }_{1,8,11}$.The deterioration in his condition while on admission can be explained by the observation on CT of a recent (acute) bleed on the left side which most likely resulted in a sudden increase in intracranial pressure and 
gave rise to the drowsiness, unsteady gait and focal seizures. When suspected, a CT or Magnetic Resonance Imaging (MRI) is necessary to confirm the diagnosis. ${ }^{10}$

The treatment for chronic subdural hematoma is mainly surgical. Conservative management is reserved for patients who are asymptomatic, high risks for surgery or who refuse surgery. ${ }^{4,10}$.It consists mainly of observation with serial CT scans or the use of steroids and angiotensin converting enzyme inhibitors $4,10,12$. Although our patient had a surgically insignificant hematoma, he was symptomatic, and was therefore managed conservatively with steroids and analgesics with good response.

\section{Conclusion}

Spontaneous chronic subdural hematoma should be suspected in older patients with chronic headache with or without other neurological features even in the absence of head trauma.

\section{References}

[1]. H.S. Wang, S.W. Kim, S.H. Kim,Chronic Subdural Hematoma in an Adolescent Girl .J Korean Neurosurg Soc 2013,53,3,201-203. http://dx.doi.org/10.3340/jkns.2013.53.3.201.**

[2]. I.W Crandon, D.Eldermire-Shearer, D. Fearon -Botha, C. Morris, K.James. A Report of Chronic Subdural Hematoma in two Elderly Patients. West Indian Med J 2007,56, 6,547-549 **

[3]. A.O Jimoh, D. A Guga, S.Danjuma, M. Mesi. Chronic Subdural Haematoma in Zaria. Orient Journal of Medicine 2015, 27,3-4 ,109-114**

[4]. J.Soleman, P.Taussky, J. Fandino and C.Muroi. Evidence-Based Treatment of Chronic Subdural Hematoma in Farid Sadaka, Tamya Quin (Eds) Traumatic Brain Injury Open Access: InTech 249-281 http://dx.doi.org/10.5772/57336.**

[5]. T. B. Rabiu Chronic subdural hematoma: A survey of neurosurgeons' practices in Nigeria, Surg Neurol Int. 2013; 4: 58.published online 2013 Apr 18. doi: 10.4103/2152-7806.110657

[6]. Bankole Olufemi Babatola, Yusuf Ayodeji Salman, Kanu Obasi Okezie, Ukponmwan Efosa, Nnadi Mathias Nnana, Arigbabu Surajudeen Oladele . Chronic Subdural Haematoma: Clinical Presentation, Surgical Treatment And Outcome At The Lagos University Teaching Hospital AJNS 2011 Vol. 30, No 1

[7]. J.C.Lin,K.Layman:Spontaneous Spinal subdural hematoma of intracranial origin presenting as back pain. J Emerg Med 2014; 47, $5,552-556 * *$

[8]. . .Luis Rafael Moscote-Salazar, Hernando Raphael Alvis-Miranda, Willen Calderón-Miranda, Gabriel Alcala-Cerra ,Andres M. Rubiano,Spontaneous chronic subdural hematoma in a young male patient: case report. Romanian Neurosurgery 2014 ,XXI ,2: 230 $-235$

[9]. J.Wang, A.Yeager, T.H. Schwartz, J.F.Fraser, G.H Westrich, Massive Spontaneous Acute-on-Chronic Subdural Hematoma Following Coumadin Administration; A Case Report JBJS Case Connector 2011,1,(2):e12 . http://dx.doi.org/10.2106/JBJS.CC.K.00021

[10]. B.B. Shehu, N.J. Ismail, Chronic subdural hematoma presenting as meningitis: A Case Report. N.J.Surg.Res 2000, 2, 1,30-32**

[11]. B.Omar,E.Mostarchid,C.Kirten etal,Chronic subdural hematoma revealed by quadriparesis. A case report. African Journal of Emergency Medicine. 2013,3,1 18-21 doi:10.1016/j.afjem 2012.07.001.**

[12]. T. Santarius, , P. J. Kirkpatrick, A.G. Kolias, , P. J. Hutchinson. Working Toward Rational and Evidence-Based Treatment of Chronic Subdural Hematoma. Clinical Neurosurgery Volume 57, 2010 Schaumburg Atlanta 112-122.** 